\title{
Prevalência e investigação das variáveis sócio-demográficas e comportamentais de pacientes com queilite actínica atendidos em um Serviço de Estomatologia da região sul do Brasil
}

\section{Prevalence and investigation of the socio-demographic and behavioral variables of patients with actinic cheilitis treated at a Stomatology Service in the southern region of Brazil]}

Juliana Tomaz Sganzerla, ${ }^{1,2,3}$, Bruna Abrunhoza dos Santos Menezes ${ }^{4}$, Mariana Sudati Rodrigues ${ }^{3}$, Mabiéli Rabelo Coelho ${ }^{4}$, Humberto Thomazi Gassen ${ }^{3,5}$, Sergio Augusto Quevedo Miguens $\mathrm{Jr}^{6}$

\section{RESUMO}

A queilite actínica é uma lesão cancerizável de caráter inflamatório que acomete o lábio, principalmente o inferior, sendo causada pela exposição prolongada e excessiva aos raios solares, ocorrendo principalmente em indivíduos do sexo masculino, de cor branca e que exercem atividades expostos ao sol. O objetivo deste estudo foi estimar a prevalência de queilite actínica entre os pacientes atendidos no ambulatório de estomatologia da Universidade Luterana do Brasil, campus Canoas, descrever suas características demográficas e avaliar os aspectos comportamentais considerados fatores de risco para o desenvolvimento de câncer bucal. A amostra foi composta por 78 prontuários de pacientes atendidos no período de 2006 a 2018. Foram coletadas as variáveis demográficas e comportamentais, sendo estas avaliadas por estatística descritiva absoluta e relativa. A idade média dos pacientes foi de 47,9 anos, sendo a maioria do sexo masculino $(53,8 \%)$ e de raça branca $(64 \%)$. A análise das variáveis comportamentais demonstrou que $34,5 \%(\mathrm{~N}=27)$ dos pacientes eram tabagistas ou extabagistas e 35,9\% ( $\mathrm{N}=28)$ não utilizavam fator de proteção labial. A alta prevalência de queilite actínica e a ausência de proteção à radiação solar indicam a necessidade de medidas preventivas para alertar a população acerca dos fatores de risco para o câncer de lábio.

Palavras-chave: Queilite. Neoplasias bucais. Odontologia. Diagnóstico.

\section{ABSTRACT}

Actinic cheilitis is a cancerous lesion which is inflammatory in nature and affects the lips, especially the lower lip. The lesion is caused by prolonged and excessive exposure to sunlight, occurring mostly in white male subjects who engage in activities exposed to the sun. This study aims at estimating the prevalence of actinic cheilitis among the patients of the stomatology clinic of the Lutheran University of Brazil, campus of Canoas, as well as describing its demographic features and assessinthe behavioral aspects considered as risk factors for the development of oral cancer. The sample consisted of 78 medical records of patients treated between 2006 and 2018. After the demographic and behavioral variables were collected, they were assessed by absolute and relative descriptive statistics. The average age of the patients was 47.9 years, the majority being male $(53.8 \%)$ and white $(64 \%)$. The analysis of the behavioral variables showed that $34,5 \%(\mathrm{~N}=27)$ of the patients were smokers or former smokers, and $35.9 \%(\mathrm{~N}=28)$ did not use any lip protection. The high prevalence of actinic cheilitis and the absence of protection to sunlight indicate the need for educational and preventive measures to warn the population about risk factors for the development of lip cancer.

Keywords: Cheilitis. Mouth Neoplasms. Dentistry. Diagnosis. 


\section{INTRODUCAO}

A queilite actínica (QA) é uma condição clínica que afeta principalmente o lábio inferior e é causada por exposição excessiva à radiação ultravioleta. É considerada uma lesão de potencial cancerizável que afeta quase predominantemente pessoas de cor de pele branca ou clara. Sua maior frequência é em homens após a quinta década de vida e que trabalham expostos ao sol. ${ }^{1,2} \mathrm{O}$ Brasil é um país que apresenta uma grande parte da população com estas características em zonas rurais e por se tratar de um país tropical que mantém grande parte da sua economia com trabalhadores frequentemente expostos à radiação solar, como agricultores, marinheiros, pescadores e trabalhadores da construção civil ${ }^{3}$, a prevenção da QA torna-se imprescindível como medida também de prevenção do câncer de lábio.

A QA apresenta duas formas clínicas, a aguda, mais comum em jovens que tiveram uma exposição recente e excessiva à radiação solar, e a crônica, que acometem indivíduos a partir da quinta década de vida com histórico de exposição prolongada e cumulativa ao sol. Ambas se caracterizam pelos lábios ressecados, com estrias e fissuras, vermelhidão, ou áreas leucoplásicas associadas ou não a áreas eritematosas, podendo ocorrer a formação de úlceras, crostas e sangramento. Geralmente é assintomática, podendo apresentar prurido em alguns casos. ${ }^{4}$

Estima-se que $17 \%$ dos casos de QA sofram transformação maligna ${ }^{5}$, no entanto, a chance de malignidade é maior em indivíduos expostos continuamente à luz solar sem proteção, sendo o fumo um fator também relacionado à etiopatogenia. ${ }^{6}$ Ainda, por ser uma lesão com crescimento lento, geralmente o paciente não percebe, o que aumenta a morbidade da doença. ${ }^{7}$

Considerada a alta prevalência e o potencial cancerizável das lesões de QA, estudos que descrevam o perfil dos indivíduos afetados e os fatores de risco associados ao desenvolvimento do câncer de lábio como o tabagismo são importantes a fim de ampliar o conhecimento científico na área e promover estratégias de controle e prevenção dessas lesões que poderão reduzir a incidência do câncer e, consequentemente, sua mortalidade. Dessa forma, o objetivo deste estudo foi verificar a prevalência de pacientes com QA referenciados ao Serviço de Estomatologia do Hospital Universitário da Universidade Luterana do Brasil (HU-ULBRA), Campus Canoas, e investigar a presença de variáveis sócio-demográficas e comportamentais consideradas na literatura científica como fatores de risco para o desenvolvimento de câncer de lábio. 


\section{MATERIAIS E METODOS}

O presente estudo do tipo transversal retrospectivo, aprovado pelo Comitê de Ética em Pesquisas da ULBRA (CEP-ULBRA), sob número de protocolo 2.751.893, teve como amostra todos os prontuários de pacientes atendidos no Serviço de Estomatologia no período entre 2006 e 2018. Como critério de elegibilidade foram selecionados todos os prontuários com diagnóstico de QA e excluídos os prontuários ilegíveis ou incompletos em relação às variáveis de interesse demográficas e/ou comportamentais. Dois examinadores treinados buscaram e selecionaram todos os prontuários na forma impressa ou digital arquivados no Serviço de Estomatologia do HU-ULBRA e utilizaram uma ficha padronizada para a coleta das variáveis sócio-demográficas (ocupação/profissão, sexo, idade e cor da pele) e comportamentais (tabagismo, consumo de bebida alcoólica e exposição ao sol). Os dados foram registrados conforme descrição dos prontuários e as informações coletadas foram compiladas para um banco de dados preparado no programa Microsoft Office Excel for Windows, versão 2010 e analisados por estatística descritiva.

\section{RESULTADOS}

Do total de 1.784 prontuários selecionados, 160 (8,9\%) apresentaram diagnóstico de lesões potencialmente cancerizáveis, sendo a QA a lesão mais prevalente com 78 casos $(4,3 \%)$. Desses, $36(46,1 \%)$ eram do sexo feminino e $42(53,8 \%)$ do sexo masculino, com idades variando entre 31 a 91 anos e idade média de 47,9 anos. A cor da pele branca foi predominante entre os casos $64 \%(n=50)$ (Tabela 1). Houve grande variabilidade no relato da profissão ou ocupação, sendo o grupo de pacientes aposentados o mais representativo (25,6\%).

A análise das variáveis comportamentais demonstrou que os hábitos de tabagismo e consumo de bebida alcoólica foram relatados no momento do primeiro exame por $10,2 \%(n=8)$ e $7,7 \%(n=6)$ dos pacientes, respectivamente. Todavia, 19 (24,3\%) declararam-se ex-tabagistas e cinco $(6,4 \%)$ ex-usuários de bebida alcoólica (Tabela 1). Em relação ao uso de fatores de fotoproteção, 30 (38,5\%) reportaram o uso de algum tipo de protetor solar labial e $100 \%$ relataram estar expostos ao sol seja por suas ocupações ou por outro motivo, embora não tenha sido possível avaliar de forma clara a exposição solar dos pacientes atendidos neste serviço. 
Tabela 1. Distribuição das variáveis sócio-demográficas e comportamentais entre os casos de queilite actínica diagnosticados no Serviço de Estomatologia do Hospital Universitário da ULBRA, campus Canoas, período de 2006 a $2018(n=78)$.

\begin{tabular}{|c|c|c|c|}
\hline $\begin{array}{c}\text { Variáveis } \\
\text { Sócio-demográficas }\end{array}$ & $\mathbf{N}(\%)$ & Variáveis Comportamentais & $\mathbf{N}(\%)$ \\
\hline Sexo & & Exposição ao sol & \\
\hline Feminino & $36(46,1)$ & $\operatorname{sim}$ & $78(100)$ \\
\hline Masculino & $42(53,8)$ & não & ---- \\
\hline Faixa Etária & & Uso de Protetor Solar & \\
\hline 30 a 50 & $15(19,2)$ & Sim & $30(38,5)$ \\
\hline 51 a 70 & $23(29,4)$ & Não & $28(35,9)$ \\
\hline$>70$ & $25(32,0)$ & $\operatorname{lgn}$ & $20(25,6)$ \\
\hline $\operatorname{lgn}$ & $15(19,2)$ & & \\
\hline Cor da Pele & & Tabagismo & \\
\hline Branca & $50(64,1)$ & Sim & $8(10,2)$ \\
\hline Parda & $5(6,4)$ & Não & $35(44,9)$ \\
\hline Negra & --- & Ex-tabagista & $19(24,3)$ \\
\hline $\operatorname{lgn}$ & $23(29,5)$ & $\operatorname{lgn}$ & $16(20,5)$ \\
\hline Ocupação/profissão & & $\begin{array}{l}\text { Consumo de bebida } \\
\text { alcoólica }\end{array}$ & \\
\hline Aposentado & $20(25,6)$ & Sim & $6(7,7)$ \\
\hline Do lar & $6(7,7)$ & Não & $47(60,2)$ \\
\hline Construção civil & $9(11,5)$ & Ex-usuário & $5(6,4)$ \\
\hline Motorista & $7(9)$ & $\lg n$ & $20(25,6)$ \\
\hline Comerciante & $4(5,1)$ & & \\
\hline Outras & $19(24,3)$ & & \\
\hline $\operatorname{lgn}$ & $13(16,6)$ & & \\
\hline
\end{tabular}

Legenda: Ign (ignorado)

\section{DISCUSSAOO}

A prevalência de QA foi de 4,3\%, o que está de acordo com os resultados relatados por outros estudos. ${ }^{2}$ Essa frequência de casos nesta população pode ser explicada pela 
localização do estado do Rio Grande do Sul em uma região de intensa radiação solar, principalmente devido ao buraco na camada de ozônio, que confere uma alta incidência de radiação ultravioleta, propiciando um número significativo de casos de QA no estado.

Em um estudo realizado em outra Instituição no mesmo estado, foi relatado 204 casos de QA numa amostra de 801 pacientes examinados, sendo a lesão de potencial cancerizável mais verificada. Ainda, quando considerado a atividade laboral daquela população, sua grande maioria eram agricultores que trabalhavam expostos ao sol sem relato do uso de fatores de proteção ${ }^{7}$, dados que corroboram com os achados do nosso estudo referente aos fatores comportamentais que propiciam o desenvolvimento das lesões de $Q A$.

A maioria dos casos de QA do presente estudo ocorreu em homens. De acordo com Lucena ${ }^{8}$ a associação com o sexo masculino ocorre devido à maior presença dos homens em atividades envolvendo exposição solar, bem como a falta de autocuidado em relação ao uso de medidas protetoras, o que facilita o surgimento das QAs. No estudo realizado por Silveira ${ }^{9}, 84,8 \%$ dos casos diagnosticados com QA eram do sexo masculino, predominantemente na quinta década de vida, e que o baixo número de mulheres acometidas talvez possa ser explicado pelo uso de protetores labiais ou de batons com filtro solar. ${ }^{9,10}$

A cor da pele branca foi a mais frequente entre os casos de QA deste estudo, assim como observado na literatura científica que é explicada pela menor proteção gerada pela melanina quando comparada a pele de cor parda ou negra. Desta forma, com menor proteção contra a ação carcinogênica dos raios ultravioletas. ${ }^{2}$

Neste estudo foi observada uma maior prevalência de QA em indivíduos com idade superior a 70 anos, estando de acordo com os dados de Lucena ${ }^{8}$, que pode sugerir uma relação com a exposição solar crônica e excessiva. No estudo de Miranda ${ }^{3}$, a média de idade dos trabalhadores da produção de cana-de-açúcar com QA foi de 37,4 anos, assim como no estudo de Silveira ${ }^{9}$ que mostrou predominância pela quinta década de vida, aproximando-se da realidade encontrada em nosso estudo, já que no presente trabalho a idade média dos trabalhadores com a lesão foi 47,9 anos.

Apenas um pequeno percentual dos pacientes $(20,5 \%)$ reportou o uso do protetor labial, o que corrobora com os achados no estudo de Cintra ${ }^{1}$, demonstrando a pouca preocupação em relação aos lábios, que também são intensamente expostos aos raios solares. 
O perfil comportamental demonstrou uma considerável porcentagem de pacientes tabagistas ou ex-tabagistas (35,8\%) que é um dos principais fatores de risco para o câncer bucal. Tal situação coloca os indivíduos em posição de risco ao desenvolvimento da doença, sendo, portanto, um grupo que deve ser acompanhado a fim de minimizar os riscos tanto de câncer bucal quanto labial. ${ }^{5}$

A avaliação da exposição solar através da atividade laboral foi prejudicada em nosso estudo devido à falta de informações e forma de preenchimento dos prontuários, visto que um grande percentual de pacientes (35,8\%) declarou ser aposentado. Contudo, não havia descrição da atividade que era desenvolvida antes da aposentadoria e que pode estar diretamente associada à exposição solar.

Embora os achados do presente estudo contribuam para um melhor entendimento da ocorrência da QA e os fatores associados a esta desordem em pacientes atendidos em um Serviço de Estomatologia de referência no diagnóstico e prevenção do câncer bucal, os resultados obtidos não permitem inferir causalidade devido a limitações do delineamento transversal utilizado. Todavia, o acompanhamento destes casos para a realização de estudos longitudinais torna-se importante para melhor entendimento dos fatores de risco, evolução de QA após controle e/ou tratamento e seus respectivos desfechos.

\section{CONCLUSOES}

A prevalência de $4,3 \%$ de casos de QA na sua maioria homens, de pele clara e com idade superior a 48 anos permitiu traçar o perfil da amostra e verificar a presença de fatores de risco para o desenvolvimento de câncer bucal, que indica a necessidade do estabelecimento de medidas educativas que orientem a população para o conhecimento sobre lesões cancerizáveis como as QAs, seus fatores etiológicos e as medidas de prevenção aos fatores de risco para o desenvolvimento do câncer bucal, tanto pela rede pública de saúde como por todos os profissionais da saúde, principalmente cirurgiõesdentistas, a orientar ao autoexame, prevenir, diagnosticar e tratar este tipo de lesão de potencial cancerizável.

\section{REFERÉNCIAS}

1. Cintra JS, Torres SCM, Silva MBF, Manhães Júnior LRC, Silva Filho JP, Junqueira JLC. Queilite actínica: estudo epidemiológico entre trabalhadores rurais do município de Piracaia - SP. Rev Assoc Paul Cir Dent. 2013; 67(2): 118-21. 
2. Arnaud RR, Soares MSM, Paiva MAF de, Figueiredo CRLV, Santos MGC dos, Lira CC. Queilite Actínica: avaliação histopatológica de 44 casos. Rev Odontol UNESP. 2014 Nov-dec; 43(6):384-389.

3. Miranda AM, Ferrari TM, Calandro TLL. Queilite actínica: aspectos clínicos e prevalência encontrados em uma população rural no interior do Brasil. Rev Saúde Pesq. $2011 ; 4(1): 67-72$.

4. Silva UH, Araújo DL, de Santana EB. Ocorrência de estomatite protética e queilite actínica diagnosticadas no centro de especialidades odontológicas da faculdade ASCES, Caruaru - PE. OdontolClín-Cient. 2011; 10(1): 79-83.

5. Neville BW, Damm DD, Allen CM, Bouquot JE. Patologia Oral e Maxillofacial. 3a edição. Rio de Janeiro: Elsevier; 2009.

6. Lúcio PSC, Barreto RC. Queilite actínica- perfil da produção científica em odontologia do Brasil nos últimos dez anos. Ver Cubana Estomatol. 2012; 49(4): 276-85.

7. Gheno JLN. Frequência de lesões bucais e sua associação com indicadores sócios demográficos, de comportamentos e estado de saúde: estudo transversal. 2011. 23 f. Trabalho de Conclusão de Curso (Graduação em Odontologia) - Faculdade de Odontologia, Universidade Federal do Rio Grande do Sul, Porto Alegre, 2011.

8. Lucena EES, Costa DCB, Silveira EJD, Lima KC. Prevalence and factors associated to actinic cheilitis in beach workers. Oral Diseases, v. 18, p. 575-579, 2012.

9. Silveira ÉJD da, Lopes MFF, Silva LMM, Ribeiro BF, Lima KC, Queiroz LMG. Lesões orais com potencial de malignização: análise clínica e morfológica de 205 casos. J. Bras. Patol. Med. Lab. 2009 Jun; 45(3): 233-238.

10. Orozco P, Vásques S, Venegas B, Rivera C. Prevalencia de queilitis actínica em trabajadores expuestos a radiación ultravioleta em Talca, Chile. Rev. Clin. Periodoncia Implantol. Rehabil. Oral. 2013 Dez; 6(3): 127-129. 